\section{Adaptive optics imaging of the outer retinal tubules in Bietti's crystalline dystrophy}

\begin{abstract}
Purpose To study the outer retinal tubules using spectral domain optical coherence tomography and adaptive optics and in patients with Bietti's crystalline dystrophy. Methods Ten eyes of five subjects from five independent families with Bietti's crystalline Dystrophy (BCD) were characterized with best-corrected visual acuity (BCVA), full-field electroretinography, and fundus autofluorescence (FAF). High-resolution images were obtained with the spectral domain optical coherence tomography (SDOCT) and adaptive optics (AO).

Results SD-OCT showed prominent outer retinal layer loss and outer retinal tubulations at the margin of outer retinal loss. AO images displayed prominent macrotubules and microtubules with characteristic features in eight out of the $\mathbf{1 0}$ eyes. Crystals were present in all ten eyes. There was a reduction in the cone count in all eyes in the area outside the outer retinal tubules (ORT).

Conclusions This study describes the morphology of the outer retinal tubules when imaged enface on the adaptive optics in patients with $B C D$. These findings provide insight into the macular structure of these patients. This may have prognostic implications and refine the study on the pathogenesis of BCD.

Eye (2016) 30, 705-712; doi:10.1038/eye.2016.22; published online 26 February 2016
\end{abstract}

\section{Introduction}

Bietti crystalline dystrophy (OMIM 210370; $\mathrm{BCD}$ ) is a rare autosomal recessive disease characterized by the presence of yellow-white crystalline retinal deposits, corneal deposits, progressive atrophy of the retinal pigment epithelium (RPE), and loss of choriocapillaris. ${ }^{1}$ Patients present with progressive night
R Battu, MC Akkali ${ }^{1}$ D Bhanushali,

P Srinivasan ${ }^{1}$, R Shetty ${ }^{1}$, TTJM Berendschot ${ }^{2}$, JSAG Schouten ${ }^{2}$ and CA Webers ${ }^{2}$ blindness; most of them are legally blind by the fifth to sixth decade of life. ${ }^{1}$ CYP4V2, the gene responsible for $\mathrm{BCD}$, codes for a member of the cytochrome p-450 family that plays a role in the metabolism of fatty acids. ${ }^{2-6}$

Outer retinal tubulations (ORT) were first described by Zweifel et $a l^{7}$ in patients with age-related macular degeneration (AMD). These appeared as hyporeflective ovoid spaces with hyper-reflective borders in the outer nuclear layer on the optical coherence tomography. They have been described in other degenerative retinal diseases like choroideremia and BCD. ${ }^{8,9}$

New imaging techniques and functional tests have evolved that are used to study patients with retinal dystrophies. These include spectral domain optical coherence tomography (SD-OCT), fundus autofluorescence (FAF), microperimetry, and adaptive optics (AO) imaging.

AO imaging is a new technology that allows direct visualization of individual rod and cone photoreceptor cells and retinal pigment epithelial (RPE) cells. ${ }^{10}$ Gocho et al ${ }^{11}$ reported an abnormal cone mosaic in the posterior pole of patients with BCD. There are no detailed description of the ORTs on the AO.

Thus, the purpose of this study was to characterize in detail the outer retinal tubulations seen in BCD using the $\mathrm{AO}$ technology. To accomplish this, we studied five patients with $\mathrm{BCD}$ using high resolution SD$\mathrm{OCT}$ and $\mathrm{AO}$ imaging.

\section{Patients and methods}

Five patients from five independent families clinically diagnosed with BCD were enrolled into the study; both eyes were included in the study. All five patients were isolated cases from non-consanguineous families. All of them were Asian Indian. The study was approved by the institutional ethics committee of Narayana
${ }^{1}$ Department of Vitreoretina, Narayana Nethralaya, Bangalore, India

${ }^{2}$ Department of Imaging, University Eye Clinic Maastricht, Maastricht, The Netherlands

Correspondence: R Battu, Department of Vitreoretina, Narayana Nethralaya, 121/C, Chord Road, Rajajinagar, I 'R' Block, Bangalore 560010, India

Tel: +91 9900265081;

Fax: +918023377329 .

E-mail: rajanibattu@gmail.com

Received: 1 November 2015 Accepted in revised form: 18 January 2016 Published online: 26 February 2016 
Nethralaya and followed the tenets of declaration of Helsinki. Informed consent for the study and publication was obtained from each patient. Every patient underwent a complete ophthalmic examination including assessment of their best-corrected visual acuity (BCVA) with the Snellen's chart, intraocular pressure measurements with the Goldmann applanation tonometer and a detailed fundus evaluation. All patients underwent full-field electroretinography (Veris systems) according to the ISCEV standards. ${ }^{12}$ Both scotopic and photopic responses were recorded. The axial length was measured in all patients using the Lens star system. They underwent further evaluation with SD-OCT and Adaptive optics imaging.

\section{SD-OCT imaging}

Imaging was performed using Heidelberg HRA2-OCT (Heidelberg retina angiograph optical coherence tomography, Heidelberg Engineering, Heidelberg, Germany). Each patient underwent a 100 ART (automatic real time) imaging in the vertical and horizontal meridians in addition to the star-scan and volume scan in the central $20^{\circ}$. Each image was analyzed for the presence of bright reflective dots and ORTs. Central foveal thickness (CFT) was measured at the center of the fovea. Using the enhanced depth-imaging mode, sub-foveal choroidal thickness (SCT) was measured from the inner border of the sclera to the outer border of the RPE vertically using the calipers of the Heidelberg reader at the same point. ${ }^{13}$

\section{Adaptive optics imaging}

Imaging was performed using the flood-illuminated AO retinal camera ( $r t x 1$, Imagine Eyes, Orsay, France). This system has been used in earlier investigations to image individual cone photoreceptors and other retinal structures. ${ }^{14-16}$ The field of imaging was $4^{\circ} \times 4^{\circ}$.

$\mathrm{AO}$ imaging sessions were conducted after dilating the pupils with $0.5 \%$ tropicamide and $10 \%$ phenylephrine hydrochloride. Aberrations induced by pupil dilation are negated by the AO system. Stable fixation was maintained by having the patient look at the system's inbuilt target and then as moved by the investigator to predetermined coordinates. The patient was instructed to fixate at $0,1,2$, and $3^{\circ}$ eccentricity along all the four quadrants: superior; inferior; nasal; and temporal retina. A video (a series of 40 frames; $4^{\circ}$ field size) was captured at each of the above retinal locations. After the acquisition, a program provided by the manufacturer correlated and averaged the captured image frames to produce a final image with a resolution of $1500 \times 1500$ pixels. ${ }^{17}$
Cone density (cones $/ \mathrm{mm}^{2}$ ) was measured at 1,2 , and $3^{\circ}$ eccentricity along all the four quadrants: superior; inferior; nasal; and temporal retina. There has been no standardized protocol on which areas to image and on the size of the sampling window to choose the region of interest (ROI). The sampling window we chose was $100 \mu \mathrm{m}$ and we placed it at specific coordinates calculated by a prefixed formula intentionally avoiding blood vessels. Our rationale was that the size of the sampling window correlates with the size of the retinal area stimulated by a Goldman size III target, so as to be able to correlate the structure and function in the future. Eccentricity was computed as the distance between the center of each window and the foveal center reference point (identified as the point with fixation coordinates: $\left.x=0^{\circ}, y=0^{\circ}\right)$. The images were captured at temporal $\left(-3^{\circ}\right.$ and $\left.0^{\circ}\right)$ superior $\left(0^{\circ}\right.$ and $\left.3^{\circ}\right)$ nasal $\left(3^{\circ}\right.$ and $\left.0^{\circ}\right)$, and inferior $\left(0^{\circ}\right.$ and $\left.-3^{\circ}\right)$. The cone counting software $\mathrm{AO}$ detect created on MATLAB by Imagine Eyes was used to process the images and calculate the cone density. Axial length was taken into consideration for calculating cone density and it was measured by non-contact biometry (IOL Master; Carl Zeiss Meditec, Jena, Germany). Both eyes of each patient were imaged.

The resulting images were stitched together using the i2k-retina software from DualAlign's i2k Retina. When i2k failed to find the position of an image, the GIMP software was used to stitch the image manually (GNU Image Manipulation Program, The GIMP Development Team; Image J, National Institute of Health, Bethesda, MD, USA).

The diameters of outer retinal tubules (ORT) were measured using an image-processing program (Image J, National Institutes of Health, Bethesda, MD, USA). The size of each pixel was typically $0.8 \mu \mathrm{m}$ when calculated at the retinal plane, and the values were adjusted for variations in the axial length of the eye. ${ }^{16,17}$

\section{Statistical analysis}

The statistical software package IBM SPSS Statistics 20 was used for analysis. All variables investigated exhibited a typical normal distribution. Mean \pm SDs are presented in the text. We used the $5 \%$ level of significance throughout our analysis.

\section{Results}

There were 3 males and 2 females with mean age of 41.4 years (range 20-60 years). The BCVA on the Snellen's chart ranged from $1 / 60$ to $6 / 6$ (Table 1 ). Fundus examination in all patients showed intraretinal, discrete, yellowish white crystals that were concentrated over the macula mainly. Crystals were not visible in the area of 


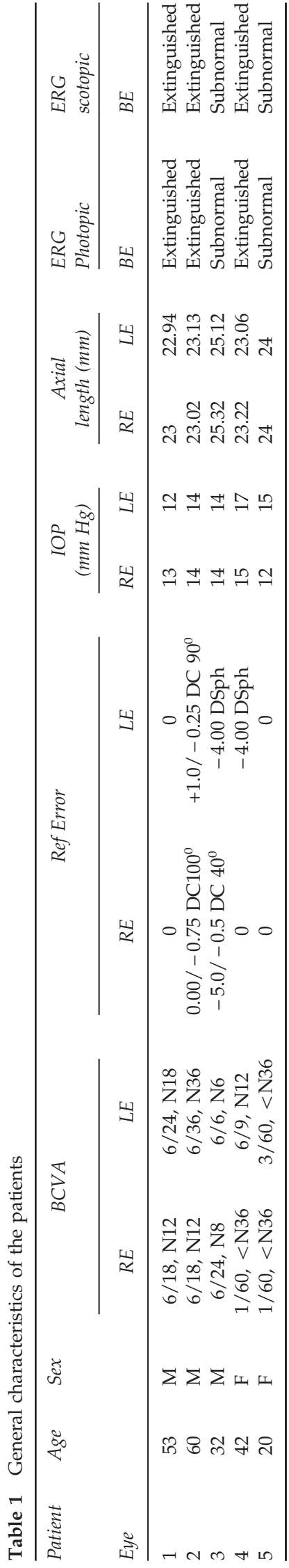

RPE atrophy (Figure 1). The older patients (patients 1 and 2) showed extensive atrophy of the choriocapillaris with fewer crystals over the macula, whereas the younger patients (patients 3, 4, and 5) showed more extensive crystals and prominent intraretinal pigmentation (Figure 1). Retinal vascular attenuation was more prominent in the older patients. Full field ERG was extinguished in three patients, and subnormal in two (Table 1). Four out of the five patients underwent evaluation of the systemic lipid profile; none of them had an abnormal lipid profile.

\section{SD-OCT imaging}

FAF showed areas of hypoautofluorescence (corresponding to RPE atrophy and loss of lipofuscin) with a hyperautofluorescent edge representing the lipofuscin-laden cells that may atrophy imminently (Figure 1). The crystalline deposits were seen more prominently on the near-infrared (NIR) images (Figure 1). None of our patients had corneal crystals.

SD-OCT in all five patients showed a complete disorganization of the outer retinal layers; the inner retinal architecture was relatively preserved. ORTs were noted in eight eyes in the outer nuclear layer at the junction of the normal and atrophic retina (Figure 2).

The average CFT was $148 \mu \mathrm{m}( \pm 73 \mu \mathrm{m})$. There was considerable foveal thinning in 3 out of the 10 eyes. Marked attenuation of the choroidal layer was noted on the enhanced depth-imaging (EDI) scans with an average SCT of $95.9 \mu \mathrm{m}( \pm 25.9 \mu \mathrm{m})$. Patient 5 demonstrated cystoid cavities and ORTs in the peripheral macula (Figure 2).

\section{AO imaging}

Cone count was decreased in all eyes. The average foveal cone count for this group was 7990 cones per $\mathrm{mm}^{2}$ ranging from 1822 to 18023 cones per $\mathrm{mm}^{2}$ area. The average foveal cone count in normal Asian eyes from our institute was 24000 cones per $\mathrm{mm}^{2}{ }^{18}$ The detailed list of cone count including fovea and para fovea are presented in Table 2.

The tubules were imaged enface on the AO. These appeared as elongated serpentine tubules with multiple branching (Figure 3). The tubules varied in size with the smaller ones (referred to as microtubules) showing more extensive and irregular branching with complex networks compared with the macrotubules. These could be clearly distinguished from the overlying blood vessels on the $\mathrm{AO}$. The tubules seem to interconnect and span the entire width of the image taken. The larger tubules seem to branch sparsely compared with the microtubules. 

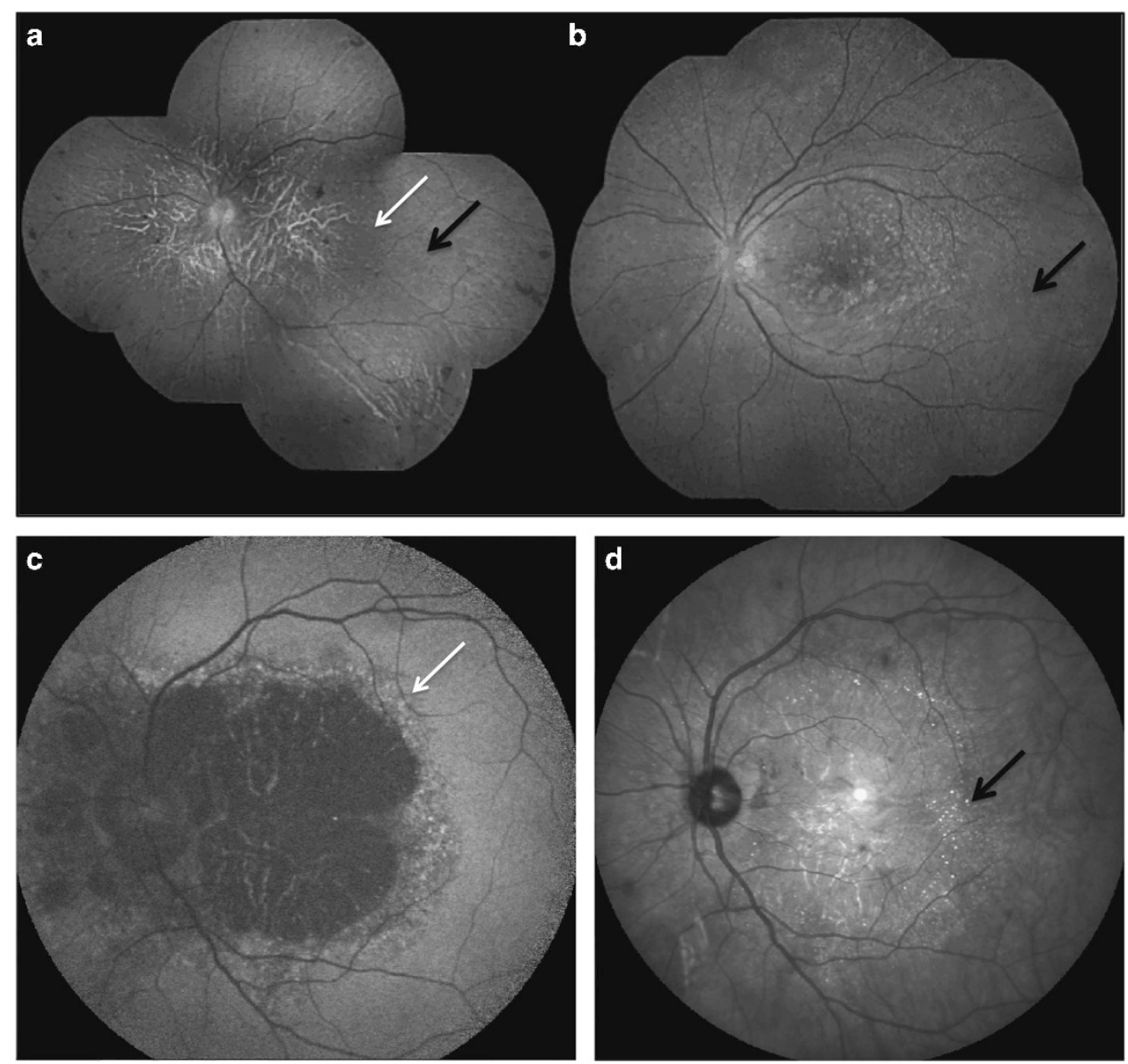

Figure 1 Fundus pictures of patients. (a) shows the fundus picture of the left eye of patient 2 (60 years old) with prominent atrophy of the retinochoroidal layers, especially over the posterior pole (white arrow). Few crystals can be noted outside this area of atrophy (black arrow). (b) The fundus picture of the left eye of a younger patient 5 (20 years old) who displays prominent crystals over the posterior pole (black arrow) with no visible areas of choroidal atrophy. (c) Fundus autofluorescence (FAF) shows the prominent area of hypoautofluorescence. Note the hyperautofluorescent edge of the lesion (white arrow). (d) The near-infrared (NIR) image of the same patient displays the retinal crystals prominently (that are not visible on the FAF) (black arrow).

Each macrotubule appeared as alternating dark and light bands spanning the width of each (Figure 3). The wall of the tubule itself appeared relatively thick with a very narrow lumen. Prominent dark patches were visible along the wall of the tubule in its entire course. The alternating bands could not be appreciated in the microtubules. The average size of the microtubules was $47 \mu \mathrm{m}( \pm 19 \mu \mathrm{m})$ and the macrotubules was $412 \mu \mathrm{m}$ $( \pm 331 \mu \mathrm{m})$.

The crystals appeared as multiple, isolated hyperreflective spots distributed irregularly. There was no specific relationship of the crystals to the tubules, although the crystals appeared larger when they were in close proximity to the tubules. The cone count was visibly reduced in the areas of the tubule formation, with very irregular cone mosaic throughout the distribution of the tubules.
In two eyes of patient 5 with extensive crystals, we were not able to identify any tubules on the AO. There were significant cystoid cavities in this patient on the SDOCT (Figure 2).

\section{Discussion}

This study describes the morphology of ORTs on AO imaging. The diagnosis of BCD was made clinically. Genotyping would have been of additional diagnostic value, but we lacked resources to conduct genotyping. Gocho et al ${ }^{11}$ described the crystals on $\mathrm{AO}$ imaging in $\mathrm{BCD}$. Striking in our study was the elongated tubules seen on $\mathrm{AO}$, in contrast to the cross section noted on the SD-OCT.

Zweifel et $a l^{7}$ proposed that degenerating photoreceptors along with the RPE and glial elements 

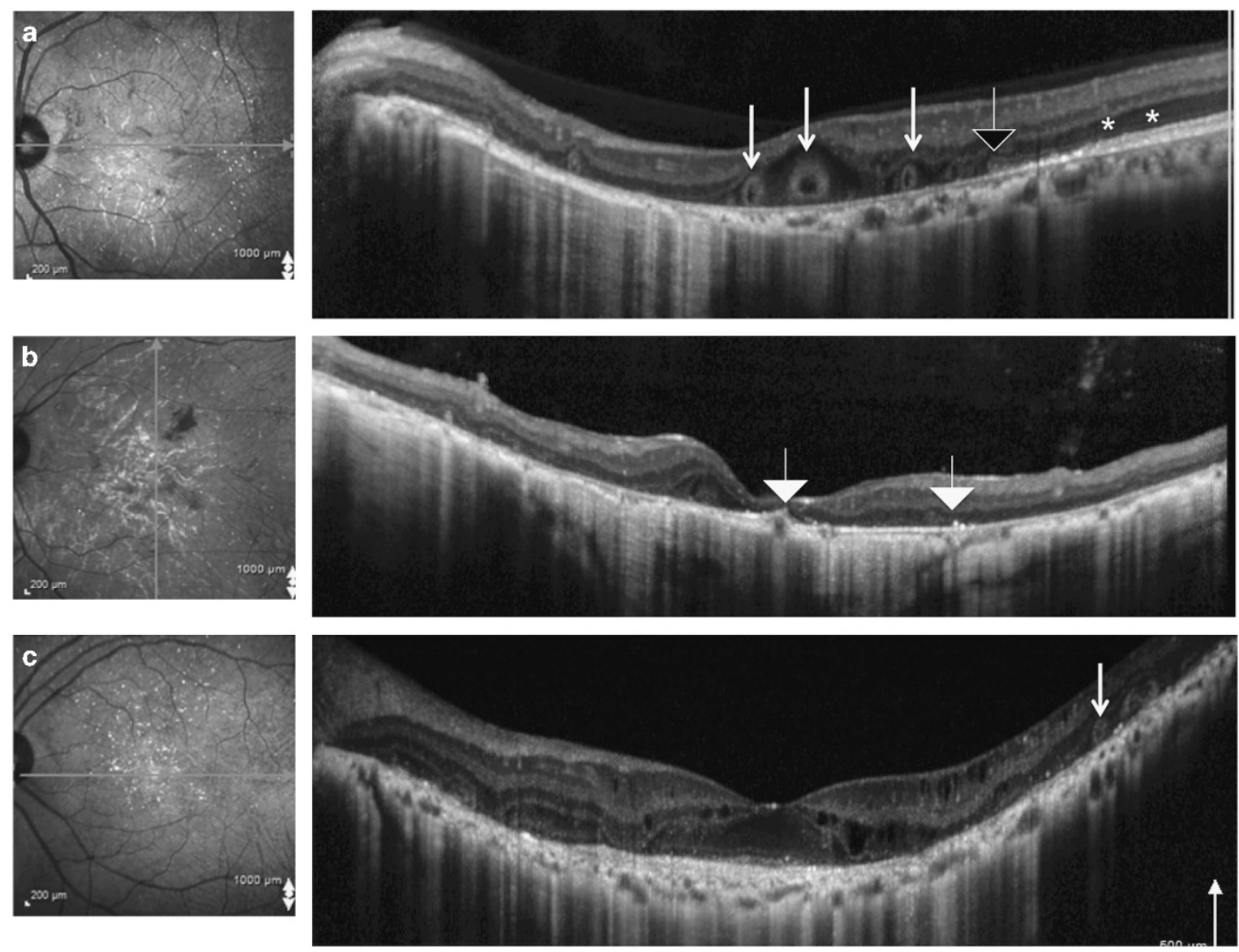

Figure 2 SD-OCT features of the patients. The pictures on the left panel throughout show the infrared images. (a) SD-OCT of the left eye of patient 2 displaying prominent ORTs (white arrows). Note that there is a significant disorganization of the outer retina in the regions of the ORTs. Beyond this region, the IS/OS layer is preserved (white stars) with a prominent scroll of the ELM at the junction (black solid arrow). The inner retinal architecture is relatively preserved. Note the severe choroidal thinning throughout. (b) The SDOCT of patient 1 . Note the crystals located on the RPE/Bruch's membrane complex (white solid arrows). (c) SD-OCT of patient 5 showing cystoid macular spaces. The ORTs are visible in the periphery of the section (white arrow).

Table 2 Details of macula on the SD-OCT and AO

\begin{tabular}{|c|c|c|c|c|c|c|c|c|}
\hline \multirow{2}{*}{$\begin{array}{l}\text { Patient } \\
\text { Eye }\end{array}$} & \multicolumn{2}{|c|}{ CFT $(\mu m)$} & \multicolumn{2}{|c|}{$S C T(\mu m)$} & \multicolumn{2}{|c|}{ Smallest ORT dia $(\mu \mathrm{m})$ on $A O$} & \multicolumn{2}{|c|}{ Largest ORT dia $(\mu \mathrm{m})$ on $A O$} \\
\hline & $R E$ & $L E$ & $R E$ & $L E$ & $R E$ & $L E$ & $R E$ & $L E$ \\
\hline 1 & 64 & 43 & 50 & 63 & 33 & 27 & 267 & 295 \\
\hline 2 & 195 & 262 & 114 & 84 & 47 & 35 & 961 & 930 \\
\hline 3 & 123 & 171 & 94 & 80 & 84 & 61 & 193 & 213 \\
\hline 4 & 64 & 171 & 96 & 119 & 32 & 53 & 185 & 253 \\
\hline 5 & 220 & 222 & 127 & 132 & - & - & - & - \\
\hline Mean \pm SD & $133.2 \pm 72.5$ & $173.8 \pm 82.5$ & $96.2 \pm 29.2$ & $95.6 \pm 28.7$ & $49 \pm 30.4$ & $44 \pm 15.7$ & $401.5 \pm 374$ & $422.7 \pm 339$ \\
\hline
\end{tabular}

came to be arranged in a tubular manner, which they called ORTs. They believed that these represented the final pathway in many retinal degenerative diseases. Photoreceptor or RPE injury lead to the loss of interdigitations and outward folding of photoreceptors at the junction of intact and disrupted ellipsoid portion of the photoreceptor inner segment initially and formation of a long, ovoid tubular complex eventually. ${ }^{7}$ Jung et al ${ }^{19}$ described that an ORT is a rearrangement of the photoreceptor layer in response to injury, in which surviving photoreceptors form new lateral connections with neighboring cells.

Histologically, ORTs were first described as interconnecting tubes containing degenerate 

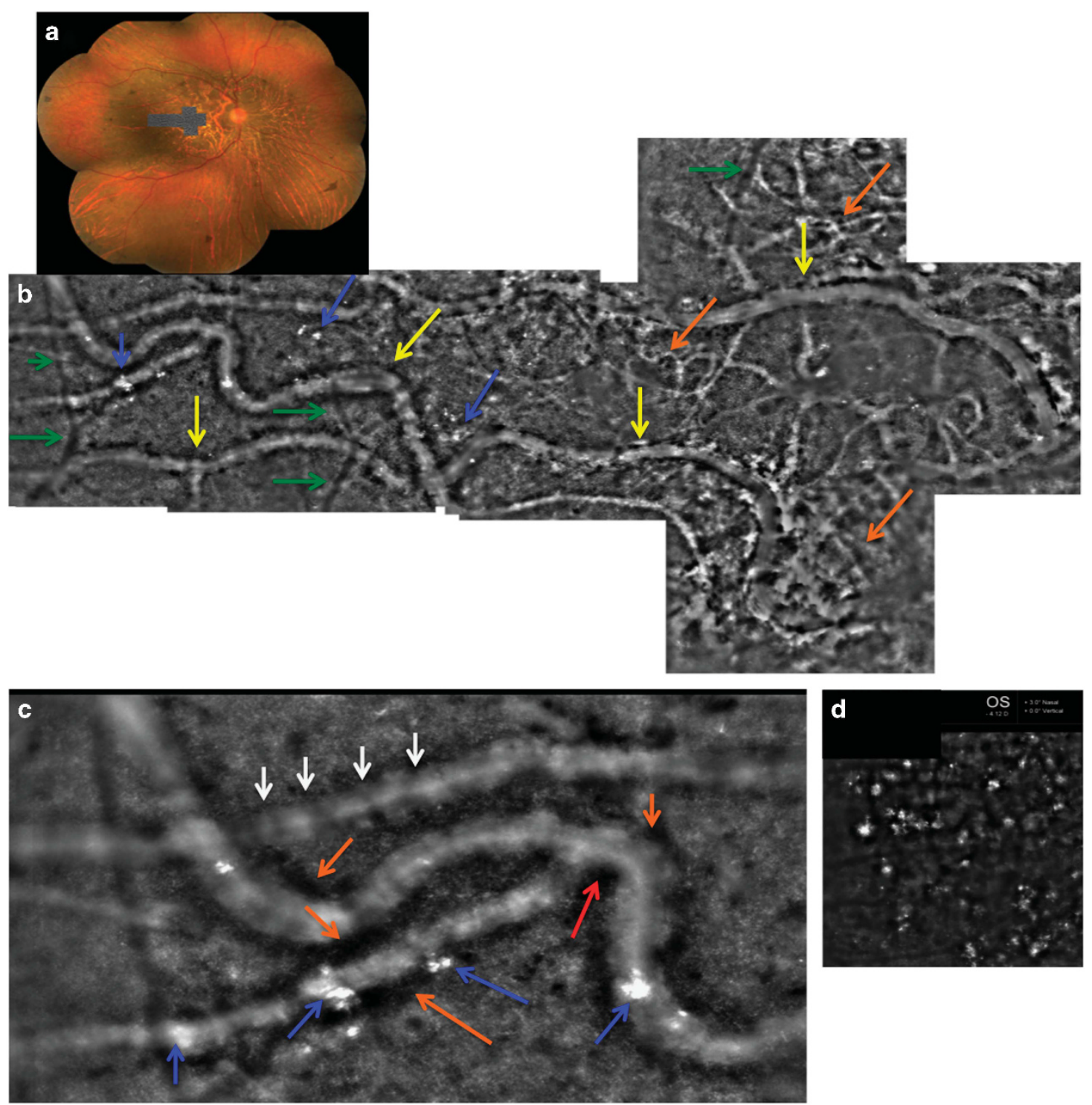

Figure 3 The overlay and the AO image of the ORTs. (a) Overlay of the AO montage on the fundus picture of patient 1. (b). Montage of the central foveal and four parafoveal $4 \times 4^{\circ}$ images obtained on the AO. Note the serpentine macro tubules (yellow arrows) that span the entire width of the image and the smaller microtubules (orange arrows) that are more complexly arranged. The blood vessels are denoted by the green arrows. The crystals are denoted by the blue arrows. (c) Further magnified view that denotes the macrotubules. Note the branching (red arrow). The tubule is marked by dark bands (white arrows) alternating with light bands throughout its length. Along the wall of the tubule are the dark patches corresponding to areas of RPE atrophy (orange arrows). The crystals themselves appeared larger when present in relation to the tubules (blue arrows) than otherwise. The cone mosaic is greatly reduced especially close to the tubules. (d) Note the prominent crystals in the left eye of patient 5 .

photoreceptors and enveloping Müller cells in AMD. ${ }^{20}$ Recently Schaal $e a^{21}$ studied the histology of ORTs in AMD. They showed that the ORTs were always present in the outer nuclear layer and largely consisted of cones lacking outer segments and/ inner segments. They defined four phases of cone degeneration histologicallynascent, mature, degenerate, and end stage ORTs. The external limiting membrane (ELM) was an important structure seen in the ORTs. ${ }^{21}$ The underlying RPE was either dysmorphic or absent. Branching of the ORTs was not seen uniformly. With time, they found shrinkage of the inner segments and involution of the ORT.

Trying to extrapolate the histological findings of Schaal et al. in AMD to the findings we noted on the adaptive optics imaging seems complicated. The alternating dark and light bands seen on the AO possibly correspond to the photoreceptors alternating with Muller cells (and other glial cells). It was hard to distinguish the ELM in the 
tubules. The cone mosaic seemed better preserved in the In the vicinity of the microtubules compared to that of the macrotubules.

We were unable to distinguish open from closed ORTs on the AO that have been described by Schaal et al. ${ }^{21}$ Histologically ORTs have been shown to involute over time with the inner segments shrinking and the mitochondria migrating through the ELM towards the cone perikaryon. ${ }^{21}$ The conglomeration of multiple microtubules may form the macrotubules. Tubule formation may be an attempt of the photoreceptors to survive in a microenvironment that is doomed to degenerate, by seeking out other photoreceptors and forming long tubules in the process.

The crystals were more clearly visible on the IR images in comparison to the FAF images. This has been described earlier. ${ }^{22}$ Gocho et al ${ }^{11}$ identified clusters of hyperreflective signals and circular spots in the $\mathrm{AO}$ images that corresponded to the crystals in three patients with BCD. They hypothesized that the circular spots were residual cone photoreceptors located over the crystals. We mostly identified dark spots along the wall of the tubules, which possibly correlated with areas of RPE atrophy.

SD-OCT demonstrates the cross section of the tubules, while the same tubules are imaged enface on the AO, appearing as long serpentine tubules. One of the inherent difficulties of imaging on the rtx 1 is in patients with cystoid macular changes. ${ }^{10}$ This may explain the apparent absence of ORTs in patient 5. Although ORTs were noted in the peripheral macula on the SD-OCT in this patient, this was outside the ROI that we imaged on the AO.

Our study had limitations. Although we attempted to correlate corresponding ORTs on the SD-OCT and AO, we were not able to obtain a good co-registration. Development of a dedicated computer algorithm may help in an accurate co-registration of the ORTs, enabling better understanding of the morphology. Accurate combining of these technologies will be able to provide high resolution, three-dimensional imaging of the retina. An ultrahigh-resolution-AO-OCT prototype may be an alternate way to image the retina to conduct larger studies. Molecular diagnosis may help establish phenotype-genotype correlations, thus helping in better understanding of the pathobiology.

This study is an attempt to describe the morphology of the outer retinal tubules on the AO. This ability to directly visualize the living retina provides an implicit advantage in diagnosing, predicting progression and monitoring retinal disease. Longitudinal studies may help observe the progression of these ORTs and correlate better histologically, providing deeper insights into the formation of these structures and therefore pathogenesis of $\mathrm{BCD}$ and other retinal degenerative diseases.

\section{Summary}

What was known before

- Outer retinal tubules are described in various retinal degenerative disorders including AMD and BCD.

- These have been noted as tubules of varying size on the SD-OCT.

- Histology of ORTs has been described in AMD.

What this study adds

- This study describes the morphology of outer retinal tubules on the Adaptive optics.

- ORTs appear as serpentine tubules of varying sizes on the AO.

- Longitudinal studies of the ORTs may help better understand the pathogenesis of Bietti's crystalline dystrophy.

\section{Conflict of interest}

The authors declare no conflict of interest.

\section{References}

1 Bietti GB. Su alcune forme atipiche o rare di degenerazione retinica (degenerazione tappetoretiniche e quadri morbosi similari). Bollettino di Oculistica 1937; 16: 1159-1244.

2 Jiao X, Munier FL, Iwata F, Hayakawa M, Kanai A, Lee J et al. Genetic linkage of Bietti crystalline corneoretinal dystrophy to chromosome 4q35. Am J Hum Genet 2000; 67: 1309-1313.

3 Li A, Jiao X, Munier FL, Schorderet DF, Yao W, Iwata F et al. Bietti crystalline corneoretinal dystrophy is caused by mutations in the novel gene CYP4V2. Am J Hum Genet 2004; 74: 817-826.

4 Lee J, Jiao X, Hejtmancik JF, Kaiser-Kupfer M, Gahl WA, Markello TC et al. The metabolism of fatty acids in human Bietti crystalline dystrophy. Invest Ophthalmol Vis Sci 2001; 42: 1707-1714.

5 Lai TY, Chu KO, Chan KP, Ng TK, Yam GH, Lam DS et al. Alterations in serum fatty acid concentrations and desaturase activities in Bietti crystalline dystrophy unaffected by CYP4V2 genotypes. Invest Ophthalmol Vis Sci 2010; 51: 1092-1097.

6 Kaiser-Kupfer MI, Chan CC, Markello TC, Crawford MA, Caruso RC, Csaky KG et al. Clinical, biochemical and pathologic correlations in Bietti's crystalline dystrophy. Am J Ophthalmol 1994; 118: 569-582.

7 Zweifel SA, Engelbert M, Laud K, Margolis R, Spaide RF, Freund KB. Outer retinal tubulation: a novel optical coherence tomography finding. Arch Ophthalmol 2009; 127: 1596-1602.

8 Goldberg NR, Greenberg JP, Laud K, Tsang S, Freund KB. Outer retinal tubulation in degenerative retinal disorders. Retina 2013; 33: 1871-1876.

9 Iriyama AY, Aihara, Yanagi Y. Outer retinal tubulation in inherited retinal degenerative disease. Retina 2013; 33: 1462-1465. 
10 Battu R, Dabir S, Khanna A, Kumar AK, Roy AS. Adaptive optics imaging of the retina. Indian J Ophthalmol 2014; 62: 60-65.

11 Gocho K, Kameya S, Akeo K, Kikuchi S, Usui A, Yamaki K et al. High-resolution imaging of patients with bietti crystalline dystrophy with CYP4V2 mutation. J Ophthalmol 2014; 2014: $1-11$.

12 Marmor MF, Fulton AB, Holder GE, Miyaki Y, Brigell M, Bach M2008Standard for clinical electroretinography (2008 update). Doc Ophthalmol 118: 69-77.

13 Margolis R, Spaide RF. A pilot study of enhanced depth imaging optical coherence tomography of the choroid in normal eyes. Am J Ophthalmol 2009; 147: 811-815.

14 Lombardo M, Lombardo G, Ducoli P, Serrao S. Adaptive optics photoreceptor imaging. Ophthalmology 2012; 119: 1498-1498 e2.

15 Lombardo M, Lombardo G, Ducoli P, Serrao S. Adaptive optics technology for high-resolution retinal imaging. Sensors (Basel) 2013; 13: 334-366.

16 Bennett A, Rudnicka AR, Edgar DF. Improvements on Littmann's method of determining the size of retinal features by fundus photography. Graefes Arch Clin Exp Ophthalmol 1994; 232: 361-367.
17 Lombardo M, Serrao S, Ducoli P, Lombardo G. Eccentricity dependent changes of density, spacing and packing arrangement of parafoveal cones. Ophthalmic Physiol Opt 2013; 33: 516-526.

18 Dabir S, Mangalesh S, Kumar KA, Kummelil MK, Sinha Roy A, Shetty $\mathrm{R}$ et al. Variations in the cone packing density with eccentricity in emmetropes. Eye (Lond) 2014; 28: 1488-1493.

19 Jung JJ, Freund KB. Long-term follow-up of outer retinal tubulation documented by eye-tracked and en face spectraldomain optical coherence tomography. Arch Ophthalmol 2012; 130: 1618-1619.

20 Curcio CA, Medeiros NE, Millican CL. Photoreceptor loss in age-related macular degeneration. Invest Ophthalmol Vis Sci 1996; 37: 1236-1249.

21 Schaal KB, Freund KB, Litts KM, Zhang Y, Messinger JD, Curcio $\mathrm{CA}$. Outer retinal tubulation in advanced age-related macular degeneration: optical coherence tomographic findings correspond to histology. Retina 2015; 35: 1-12.

22 Halford S, Liew G, Mackay DS, Sergouniotis PI, Holt R, Broadgate $\mathrm{S}$ et al. Detailed phenotypic and genotypic characterization of bietti crystalline dystrophy. Ophthalmology 2014; 121: 1174-1184. 